\title{
Tangible NURBS-curve Manipulation Techniques Using Graspable Handles on a Large Display
}

\author{
Seok-Hyung Bae ${ }^{1}$, Takahiro Kobayashi ${ }^{2}$, Ryugo Kijima ${ }^{1}$, and Won-Sup Kim ${ }^{3}$ \\ ${ }^{1}$ Virtual System Laboratory \\ ${ }^{2}$ Institute of Advanced Media Arts \\ Gifu University \\ and Sciences \\ 1-1 Yanagido, Gifu \\ 501-1193 Japan \\ 3-95 Ryoke-cho, Ogaki, Gifu \\ 503-0014 Japan \\ bae|kijima@vsl.gifu-u.ac.jp \\ kobayasi@iamas.ac.jp \\ ${ }^{3}$ Dept. of Industrial Design \\ KAIST, 373-1 Guseong-dong, \\ Yuseong-gu, Daejeon \\ 305-701 Republic of Korea \\ iron@mail.kaist.ac.kr
}

\begin{abstract}
This paper presents tangible interaction techniques for fine-tuning one-to-one scale NURBS curves on a large display for automotive design. We developed a new graspable handle with a transparent groove that allows designers to manipulate virtual curves on a display screen directly. The use of the proposed handle leads naturally to a rich vocabulary of terms describing interaction techniques that reflect existing shape-styling methods. A user test raised various issues related to the graspable user interface, two-handed input, and large-display interaction.
\end{abstract}

Categories and Subject Descriptors: H.5.2 [User Interfaces]: Input devices and strategies, Interaction styles; I.3.6 [Methodology and Techniques]: Interaction Techniques; I.3.5 [Computational Geometry and Object Modeling]: Splines; J.6 [Computer-Aided Engineering]: Computer-aided design (CAD)

Additional Keywords and Phrases: Automotive design, NURBS-curve manipulation, large display, graspable handle, graspable user interface, two-handed input

\section{INTRODUCTION}

The large-scale representation of cars plays a crucial role in automotive design because the emotional response to the final product may depend on the scale [9]. Therefore, automotive designers have developed specialized large-scale styling methods, such as tape drawing and clay modeling. However, the analogue characteristics of these traditional methods involve serious difficulties in effective management and the reuse of styling data, and constitute a major obstacle to the successful digitalization of the whole automotive-design process.

Permission to make digital or hard copies of all or part of this work for personal or classroom use is granted without fee provided that copies are not made or distributed for profit or commercial advantage and that copies bear this notice and the full citation on the first page. To copy otherwise, or republish, to post on servers or to redistribute to lists, requires prior specific permission and/or a fee.

UIST '04, October 24-27, 2004, Santa Fe, New Mexico, USA.

Copyright (C) 2004 ACM 1-58113-957-8/04/0010 . . \$5.00.
In an effort to overcome these problems, Balakrishnan et al. [4] developed digital tape drawing, which is an electronic analogue of the physical tape drawing used in automotive design studios for creating large-scale straight lines and smooth curves on an upright surface with adhesive photographic tape. The digital technique corrected many drawbacks of the analogue method and subsequent studies of this technique led researchers to new design possibilities $[13,14]$. However, digital tape drawing does not have delicate curve modification functions, because its main purpose is to create new curves quickly and flexibly, not to fine-tune existing curves. From the viewpoint of ideal digital-design flow, in which all styling data are electronically transferred to each design step [3], digital techniques are needed that can bridge the gap between the early styling and engineering design stages by allowing designers to refine curve models on a large display.

This paper describes a new method of modifying large NURBS curves precisely at full scale (Fig. 1). We present a cylindrical graspable handle with a transparent groove to interact with virtual curves on the display screen in a more tangible manner. The proposed handle leads to intuitive two-handed interaction techniques that correspond to existing styling methods, so that the designer can manipulate NURBS curves naturally and effectively.

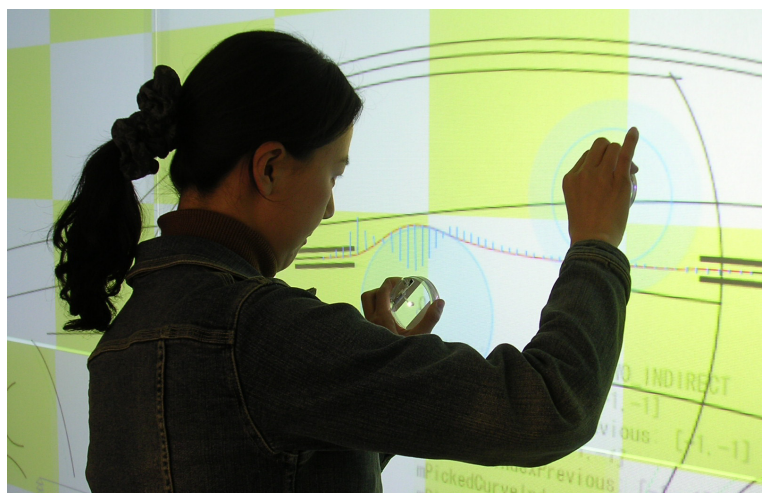

Figure 1: Tangible NURBS-curve manipulation on a large display 
This paper is organized as follows. First, related work is summarized. Then, the design goals of our digital system are set, based on an analysis of NURBS-curve manipulation tasks and the large-wall working environment. Third, a hardware system and interaction techniques are highlighted to satisfy the design goals. Fourth, user comments and related issues are discussed. Finally, conclusions and a description of future work close the paper.

\section{RELATED WORK}

A great deal of research has been dedicated to the task of transforming traditional styling methods into digital media. Designers' sketching techniques have been analyzed and digitalized $[1,2,6]$. These pen-based drawing interfaces can increase the designer's performance markedly in the sketchbook-sized interaction environment, but are not appropriate for drawing large-sized curves on wall displays directly. A French curve, a type of predefined curve template, has been implemented in graphics software [22,28]. The user can create a new curve or edit an existing curve using an electronic pen device with any one of a set of digital French curves. In this paper, we suggest curve-editing techniques that use virtual scraping similar to those performed with a digital French curve. However, the user's hand motions control our shaping template flexibly, so there is no need to obtain and select a particular template of the required shape. As mentioned in the introduction, digital tape drawing [4] is a standard study in the digitalization of an existing analogue styling method. We expect great synergy from combining the flexible curve creation ability of digital tape drawing with our sophisticated curve-modification techniques for an effective digital-design environment. In addition, an idiosyncratic method for creating and modifying curves by bending and twisting a strip-type input device exists $[5,15]$. Such a method gives the user high tangibility with a physical curve object that corresponds to a virtual curve widget on a computer screen, but is not compatible with one-to-one scale curve work.

The graspable user interface (graspable UI) $[11,29]$ is one of the most important concepts in our study. The graspable UI improves the communication between the user and computer by using specialized, context-sensitive input devices (physical handles) with which the user can tangibly manipulate virtual objects. Furthermore, the graspable UI encourages two-handed interaction and helps the user to use spatial reasoning skills fully, enabling the development of good interaction techniques for artistic work. In our study, we tried to adopt the advantages of a graspable UI by introducing a specialized graspable handle that is appropriate for large-curve manipulation.

Many studies have shown that certain tasks performed with two-handed input are superior to those done with one-handed input $[8,20,24]$. Specifically, Owen et al. [24] proved empirically that two-handed input is effective when editing curves. Guiard [16] suggested the kinematic chain (KC) model of skilled two-handed motion, where the dominant (D) and non-dominant (ND) hands have asymmetric roles. The principles of the $\mathrm{KC}$ model are as follows: (1) ND defines the reference frame for motion of $\mathrm{D}$; (2) the motion of D has a higher temporal and spatial frequency than that of ND; and (3) ND starts to move before D. Since two-handed interaction techniques following these rules tend to be natural and effective [20], we devised curve-manipulating operations that obeyed these principles.

A highly interactive task on a computer display requires a comfortable working environment. Therefore, much research has examined how to provide the user with enough space for the main task without disturbing the user's visual attention $[7,18,21,22]$. In this paper, we adopt many valuable ideas from previous studies to develop fluid interaction techniques in a large-display environment.

\section{LARGE-SCALE CURVE MANIPULATION}

In this section, we examine the nature of a NURBS-curve manipulation task that is currently performed using geometric modeling software and the large-wall interaction of traditional tape drawing. Based on these observations, we identify several design goals for a successful digital interaction system, then, suggest design solutions to achieve these goals.

\section{Analysis}

NURBS-Curve Manipulation. The large-scale NURBS curves used in automotive design are of very high quality in both engineering and aesthetic terms. To work with such curves, users must understand the basic underlying mathematics. Existing geometric modeling software offers a wide range of curve-modeling functions, from comparatively simple to very complex. In many cases, the position and tangent of a point on a curve, which provide clear visual geometric information, are often referred to and modified simultaneously.

Large-wall Interaction. We decided that the working environment of our digital system would be similar to that of physical tape drawing. The drawing surface is upright and large. Physical feedback plays an important role in achieving high-quality artwork; tape artists press their hands on the wall and lean against the wall for stability when handling the tape. The designer works standing up and keeps walking; the designer performs curve-creating operations close to the wall, but frequent evaluation from a distance is necessary. Arm-length interaction is physically demanding on the entire body. Sometimes, more than one person work together on the same wall.

Designer's Preference. Designers are generally familiar with traditional styling media, and prefer an artistic working style that does not require mathematical understanding.

\section{Design Goals}

Based on these observations, we set the following five goals for our hardware system and interaction techniques:

- Tangibility: Designers must handle the curves displayed on the screen directly to show their artistic talent fully. 
- Precise shape control: Accurate management of the geometry of the curves is essential in high-quality curve design.

- Rich vocabulary: Various NURBS-curve operations should be provided so that designers can reflect their specific intentions exactly.

- Fluid interaction: Highly interactive work on a large-wall display requires fluid, seamless interaction for the designer's comfort and operational efficiency.

- Acceptable wall display: The display screen has to be big enough to represent the large curves of vehicles with sufficient image resolution. In addition, such a display system should have a rigid surface for accurate curve manipulation.

\section{Solutions}

Hardware. In our application, where controlling geometry precisely is the main task, finger motions or hand gestures [32] are difficult to use because of ambiguity in delimitating actions [11]. Therefore, we developed a graspable handle $(\mathrm{GH})$ associated with the action of seizing a curve. Its unique shape with a transparent groove makes it possible to handle both the position and tangent information for a given curve simultaneously. Our GH includes one discrete controller that the designer uses to enter decision commands to confirm a specific action. Unlike small-display interactions [5,15], foot pedals are not suitable for large-display interaction. Based on user feedback about digital tape drawing [4], wireless operation of the $\mathrm{GH}$ is an essential feature, because a wire would obstruct the designer's two-handed motion during curve handling. A tiled, back-projection display configuration fits our design goals. If the display screen is rigid, the designer can receive clear tactile feedback from it.

Interaction Techniques. We designed two-handed interaction techniques that follow the $\mathrm{KC}$ model [16] by reflecting traditional styling techniques and borrowing ideas for fluid interaction on large displays $[7,8,21,22]$.

Table 1 shows how the suggested design solutions satisfy the design goals. We identify the first four solutions as $\mathrm{GH}$ design guidelines.

Table 1: Mapping solutions onto design goals

\begin{tabular}{|l|l|l|l|l|l|}
\hline \multirow{2}{*}{ Solutions } & \multicolumn{5}{|c|}{ Design Goals } \\
\hline & T & P & R & F & W \\
\hline GH w/ curve-grabbing metaphor & $\checkmark$ & & $\checkmark$ & & \\
\hline GH w/ position/orientation indicator & & $\checkmark$ & $\checkmark$ & & \\
\hline GH w/ discrete controller & & $\checkmark$ & $\checkmark$ & & \\
\hline GH w/o wire & $\checkmark$ & & & $\checkmark$ & \\
\hline Tiled rear-projection display & & $\checkmark$ & $\checkmark$ & & $\checkmark$ \\
\hline Rigid display screen & & $\checkmark$ & & & $\checkmark$ \\
\hline Two-handed interaction & $\checkmark$ & $\checkmark$ & $\checkmark$ & $\checkmark$ & \\
\hline Traditional styling techniques & $\checkmark$ & $\checkmark$ & $\checkmark$ & $\checkmark$ & \\
\hline Fluid interaction ideas & $\checkmark$ & & $\checkmark$ & $\checkmark$ & $\checkmark$ \\
\hline
\end{tabular}

T: Tangibility, P: Precise shape control, R: Rich vocabulary, F: Fluid interaction, W: acceptable Wall display

\section{HARDWARE SYSTEM}

The proposed hardware system consists of two GHs with which the designer can hold and move a curve, and a large perceptual wall that can interpret the designer's gestures with the GHs, as shown in Fig. 2.

\section{Large Perceptual Wall System}

Based on the design goals and solutions in the previous section, we determined that machine vision techniques $[10,26,29,31]$ were more appropriate for tracking the user's action with the GHs than using magnetic sensors with a wire. As shown in Fig. 2, we chose a HoloWall-type perceptual wall configuration [26] that uses IR-LEDs and an IR filter.

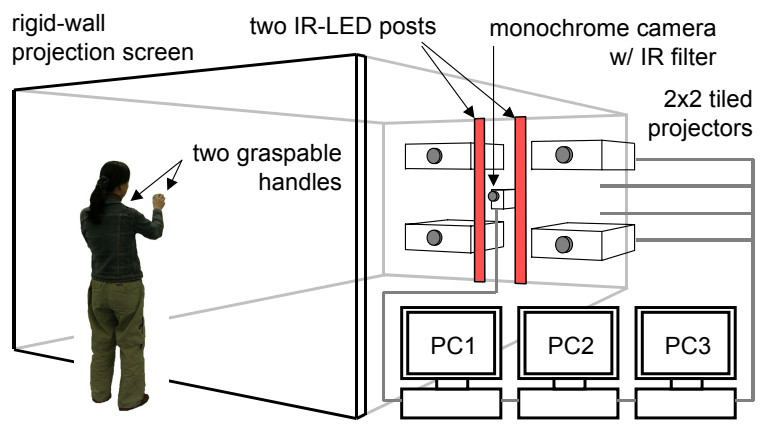

Figure 2: Hardware system configuration

The projection screen was designed for showing the true size of small passenger vehicles $(4,400 \mathrm{~mm} \times 2,300 \mathrm{~mm}(\mathrm{~W} \times \mathrm{H}))$, and was constructed of transparent acryl $(10 \mathrm{~mm}(\mathrm{~T}))$ and a diffusive sheet. By tiling four LCD projectors (SANYO LP-W1000, producing $1,366 \times 768$ pixels $(\mathrm{W} \times \mathrm{H}))$ with a short lens (LNS-W31), we obtained a resultant image with a resolution of 2,048 $\times 1,075$ pixels $(\mathrm{W} \times \mathrm{H})$. One IEEE1394 monochrome digital camera (VIEW PLUS Scorpion, 1,280 $\times 960$ pixels $(\mathrm{W} \times \mathrm{H}))$ with an IR filter captures the rear of the screen. Twenty-four IR-LED units (each with 56 IR-LEDs) are positioned near the camera. The system uses three PCs: one to capture images (Intel Pentium 4, $1 \mathrm{GHz}, 384 \mathrm{MB}$ RAM), one to run the application program for NURBS-curve manipulation (Intel Pentium 4, $1 \mathrm{GHz}, 1,024$ MB RAM), and one to generate output images (Intel Pentium 4, 3 GHz, 1,024 MB RAM).

\section{Graspable Handles}

We created a new GH made of acryl with a transparent groove that satisfied the four design guidelines for our $\mathrm{GH}$ (Fig. 3(a) and (b)). The distinct pattern on the bottom face, which was created by attaching retro-reflective sheets, gives the user sure physical control when holding curves and manipulating position and tangent information, and allows a rich vocabulary describing intuitive curve operations. Furthermore, a finger motion, in which the user touches the $\mathrm{GH}$ with the index finger and blocks the transparent slit (Fig. $3(c)$ ), functions as a discrete controller. For stable detection of the finger motion, we mounted a simple electric circuit with a small battery on our GH. If the user connects the two wires around the wall of our GH by touching them with the 
middle finger, the IR-LED emits an infrared light on the bottom (Fig. 3(d)). This so-called "electric fingering" motion generates a consistent silhouette of the fingered $\mathrm{GH}$ on the screen. Electric fingering makes image processing much easier than with the original fingering motion. Note that an LED lights up simultaneously with the IR-LED to tell the user that the fingering was successful visually.

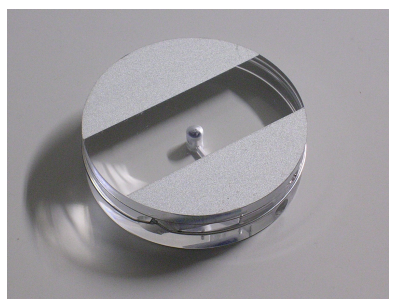

(a)

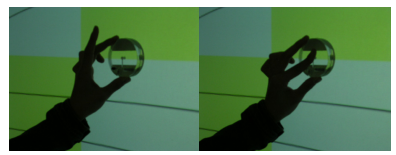

(c)

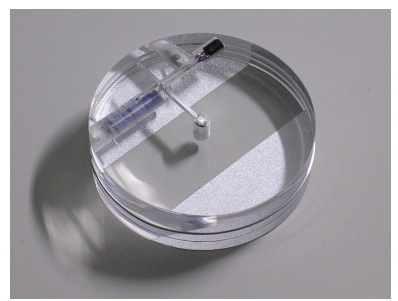

(b)

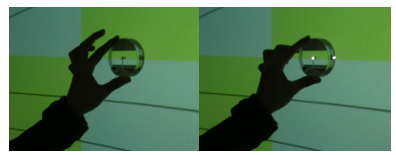

(d)
Figure 3: Graspable handle: (a) bottom face, (b) top face, (c) fingering on groove, and (d) electric fingering

To provide the user with the same grip conditions regardless of its orientation, our GHs are cylindrical $(70 \mathrm{~mm} \times 20 \mathrm{~mm}$ $(\mathrm{D} \times \mathrm{T})$, with a 20 -mm-wide see-through groove) and have a wire-type electrical switch instead of buttons.

\section{INTERACTION TECHNIQUES}

In this section, we describe various interaction techniques used with our GHs, from basic operations to complicated NURBS-curve modification.

\section{Basic Operations with the Graspable Handle}

Our GH has three continuous state variables (CSV), $\{\mathrm{x}, \mathrm{y}$, angle $\}$ and two discrete state variables (DSV), \{onScreenFlag, fingeringFlag\}. The CSVs are valid only when the $\mathrm{GH}$ is on the display screen. The onScreenFlag can be toggled when the GH silhouette appears or disappears on the image captured by the camera, and the fingeringFlag is based on the silhouette shape of the GH.

Considering the two DSVs, our GH has four discrete states \{up, suspend, hover, down\}, as depicted in Fig. 4. The GH can be moved between the four state layers using a set of discrete actions: \{place, remove, press, release, (press+place), and (press+remove) $\}$, where (press+place) and (press+remove) are the actions of placing and removing the $\mathrm{GH}$ while fingering, respectively. In fact, the computer cannot distinguish suspend from up. However, we chose a virtual state-action model with separate suspend and up layers for expressing (press+place) and (press+remove). In the hover and down layers, continuous actions \{slide, drag\} are possible. Fig. 5(a) and (b) shows the schematic notation for continuous and discrete actions with our $\mathrm{GH}$.

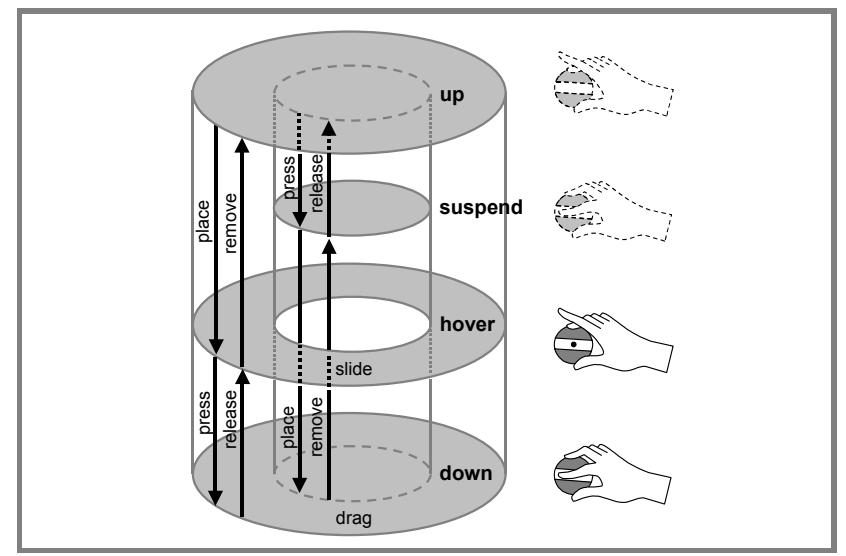

Figure 4: State-action cylinder of the graspable handle

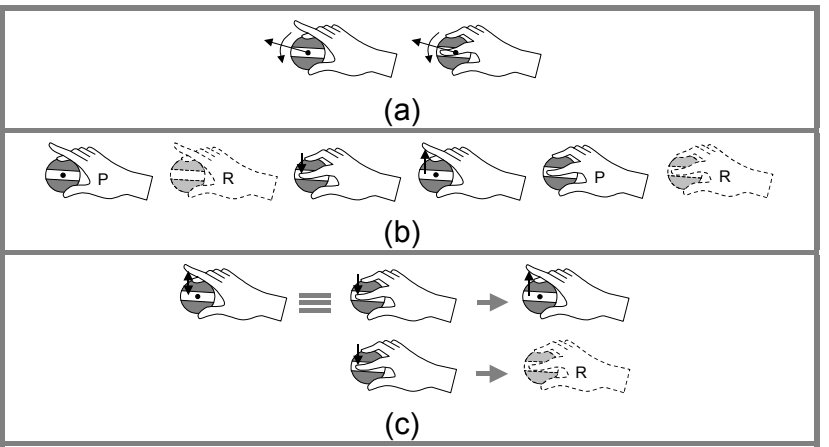

Figure 5: Schematic notation for (a) continuous actions \{slide, drag\}, (b) discrete actions \{place, remove, press, release, (press+place), (press+remove)\}, and (c) click

"Click" is defined as successive press and release actions. However, a basic premise of the graspable UI, i.e., that a chosen object becomes deselected when the GH is removed from the screen, requires additional consideration. In our study, the press and remove combination is also treated as a click (Fig. 5(c)). With the click action, the posture information of the $\mathrm{GH}$ when pressed is stored for upcoming curve operations; release or remove means the action is completed.

\section{Basic On-screen Gestures with the Graspable Handles}

The gestures used with our GHs are categorized into three types:

- Groove gestures \{half-hold, full-hold, half-cut, full-cut\}: The groove pattern on the bottom of our $\mathrm{GH}$ is used to actively express the user's intentions with respect to curves. Hold gestures, putting a curve between the groove of our GH (Fig. 6(a) and (b)), are a powerful metaphor for grasping long, thin objects. Half-hold is interpreted as seizing a curve loosely (Fig. 6(a)). Therefore, it cannot be used to affect the position or shape of the curve. Conversely, full-hold means seizing a curve tightly, while keeping the orientation of the curve at the holding point fixed to that of the $\mathrm{GH}$, like biting a beam with a 
mechanical vise (Fig. 6(b)). Similarly, cut gestures represent physical cutting or conceptual discontinuity (Fig. 6(c) and (d)). The half-cut gesture can be interpreted as aiming before decisive cutting. As in our physical world, it is possible to slide the $\mathrm{GH}$ over a curve to find a final cutting point while maintaining the half-cut gesture. Naturally, full-cut implies a decisive cutting action. These clear meanings of the groove gestures both help the user to understand our interaction techniques easily and enhance the performance of curve operations.

- Stroke gestures \{erase, rub\}: By identifying the trajectory of the GH on the screen, the computer can interpret the user's intention. Erase [19] and rub are typical pre-defined trajectory patterns (Fig. 6(e) and (f)).

- Inter-GH gestures \{collide, draw : It is possible to convey special meanings from the relative movements of two GHs. Collide and draw are gestures used to create a new curve object (Fig. 6(g) and (h)).

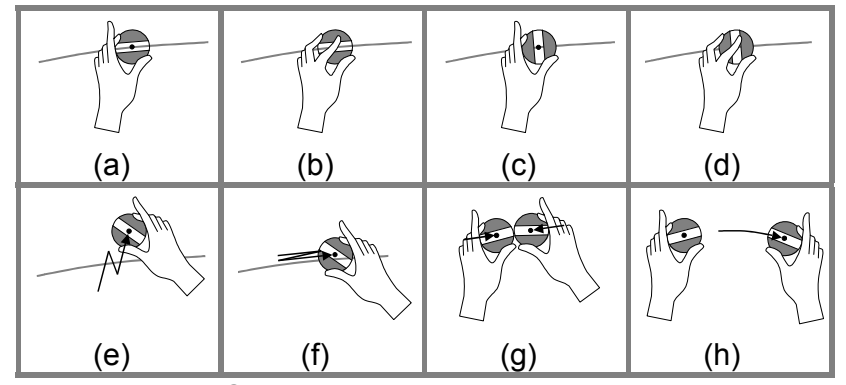

Figure 6: Gestures with graspable handles: (a) half-hold, (b) full-hold, (c) half-cut, (d) full-cut, (e) erase, (f) rub, (g) collide, and (h) draw

\section{Simple NURBS-curve Manipulations}

This subsection presents comparatively simple curve-operation techniques that are derived from the basic $\mathrm{GH}$ actions and gestures.

- Select and deselect: The user can select a curve by using the half-hold gesture (Fig. 7(a)). This is a more concrete, specialized selection concept for curve handling than the simple put-and-pick concept of a general graspable UI [11]. If the state of the $\mathrm{GH}$ changes to a simple hover state (not half-hold), or if the $\mathrm{GH}$ is removed from the screen, the chosen curve is deselected.

- Split: While half-holding a curve with the non-dominant hand (ND), the user seeks a cutting point by using the half-cut gesture with the dominant hand (D) and then divides (full-cut) the curve in two. Releasing or removing the $\mathrm{GH}$ grasped with the dominant hand $(\mathrm{GH}(\mathrm{D}))$ completes the split operation (Fig. 7(b)). As previously mentioned, this sequential action of $\mathrm{D}$ represents a single click action. Note that it is possible to do successive split operations if the ND continues to hold the curve.

- One-handed rigid-body transform: By seizing (full-hold) a selected curve tightly, the user can move it (Fig. 7(c)). In our early research on tangible NURBS-curve manipulation, there was no fingering concept and no mechanical switch on the GH; there was only full-hold without half-hold. Therefore, we would inadvertently move a curve while sliding the GH to reach the position and orientation we wanted. To solve this problem, we tried several things. First, we considered a two-handed moving method; however, the tangent condition imposed by the full-hold gesture could not be maintained during rotational transformation. Second, we discussed a dwell-time interface, in which a short time interval was inserted before the chosen curve was ready to move, but we discarded this idea because waiting might frustrate the user. Third, we considered different thresholds for selecting and moving a curve. However, unlike screen zoom and pan purposes [14], it was difficult to distinguish between selecting a curve and a small amount of sophisticated curve moving. In the end, we had to introduce a discrete controller using fingering.

- Two-handed rigid-body transform: The user can move a curve by means of two-handed motion, while half-holding a curve with the $\mathrm{GH}$ held by the non-dominant hand (GH(ND)) and GH(D) down (Fig. 7(d)). This two-handed rigid-body transform is a simultaneous translation and rotation around $\mathrm{GH}(\mathrm{ND})$. Although the tangent direction of the curve at the pivot does not match the orientation of $\mathrm{GH}(\mathrm{ND})$, our premise of the half-hold gesture, loosely holding a curve, is maintained. This curve-moving technique with two hands is particularly useful when delicate curve repositioning is necessary, because it helps the machine vision program to determine the change in the rotational transformation accurately.

- Scale: A mixed scaling operation including translation and rotation is possible by dragging two GHs at the same time, while full-holding a curve with GH(ND) and simply placing GH(D) on the background (Fig. 7(e)). This compound task is a typical anchor/actuator interaction $[11,22,29]$. Compared with the two-handed rigid-body transformation, both the position and tangent conditions are kept at the pivot while the curve is being deformed.

- Delete: The user can delete a curve half-held with GH(ND) using an N-shaped stroke [19] with GH(D) over the selected curve (Fig. 7(f))

\section{NURBS-curve Deformations}

Delicate partial deformations of a NURBS curve involve three steps: (1) imposing deform range and boundary conditions on a curve, (2) reshaping the curve with one- or two-handed motions, and (3) fixing the curve resulting from the deformations.

Step 1. Impose Boundary Conditions. The area to deform and the boundary condition at each endpoint are defined with two-handed motions. The fixed-end (Fixed-BC) and free-end (Free-BC) boundary conditions correspond to hold and cut gestures with GH(D), respectively (Fig. 8(a) and (b)). At the point where Free-BC is defined, the curve is split. Therefore, Free-BC cannot be defined at both ends. As an exception, it is permissible to define the second boundary condition by clicking GH(ND) for Fixed-BC at both ends for operational fluidity (Fig. 8(c)). 


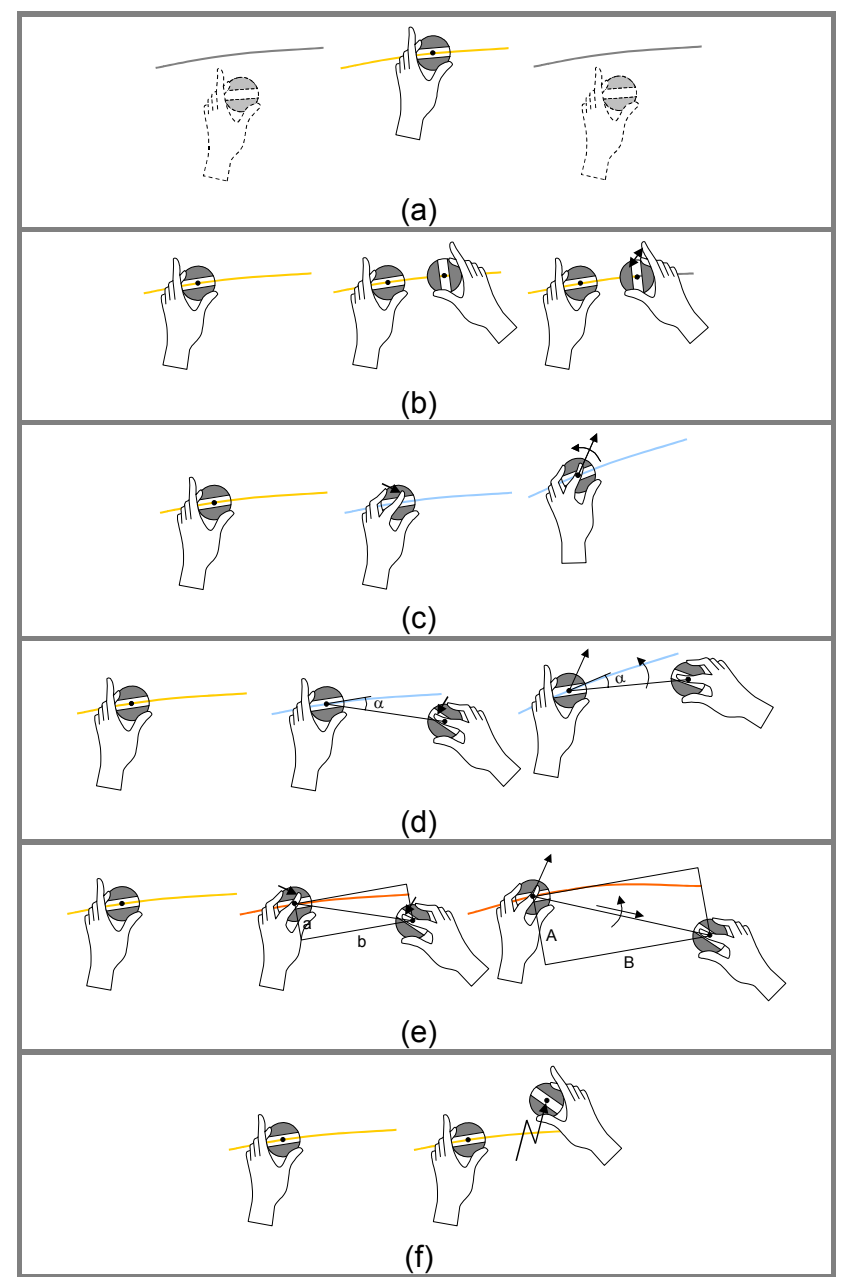

Figure 7: Simple NURBS-curve manipulations: (a) select and deselect, (b) split, (c) one-handed rigid-body transform, (d) two-handed rigid-body transform, (e) scale, and (f) delete

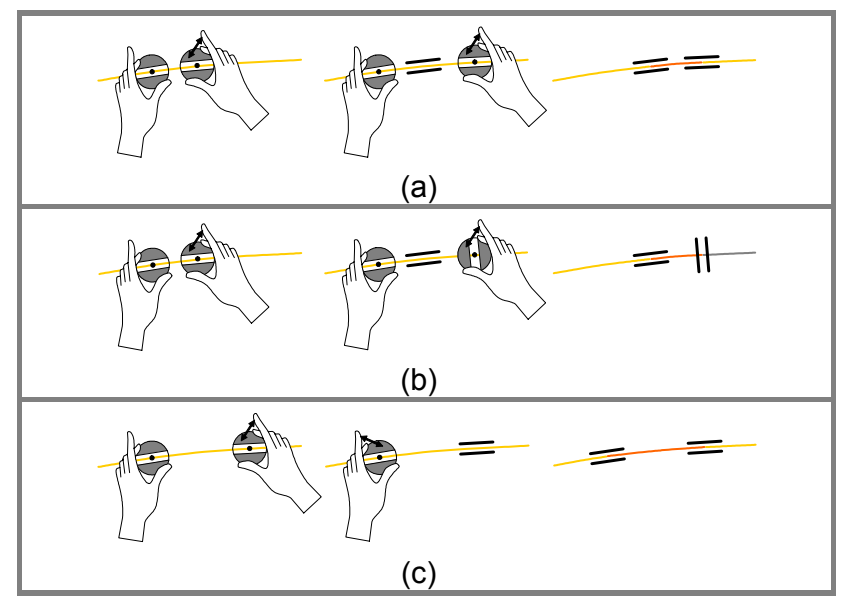

Figure 8: Imposing the boundary conditions

Step 2. Deform. Once the deform range and boundary conditions are assigned, the designer can reshape the curve using various one- and two-handed curve reshaping techniques that parallel traditional methods, such as editing with French and flexible curves, and scraping with a sweep [33] in $3 \mathrm{D}$ clay modeling.

- One-handed direct deform: The designer can seize a curve by full-holding with one GH and then deform the curve by dragging it (Fig. 9(a)). The curve is changed so that the corresponding held point keeps the position and orientation of the $\mathrm{GH}$.

- One-handed indirect deform: First, the designer places a $\mathrm{GH}$ on the screen where there is no curve beneath it. Then, he or she can deform a curve by pushing the GH against it (Fig. 9(b)). When the distance from the GH to the curve $\left(R_{C}\right)$ is between the radius of the $G H\left(R_{H}\right)$ and a pre-defined acting range $\left(R_{A}\right)$, curve deformation occurs $\left(R_{H}<R_{C}<R_{A}\right.$, refer to Fig. 16(b)). If the $\mathrm{GH}$ reaches the curve, the change stops.

- Two-handed deform (direct/indirect/mixed): Like manipulating a flexible curve, the designer can operate the curve in a variety of ways using two GHs (Fig. 10).

- Deform with a flexible template: Using successive collide and draw gestures, the designer can generate a flexible template (FT), which is modeled as a cubic Bezier curve mathematically (Fig. 11). If the angle difference of the two GHs becomes less than a pre-defined value, the length of the FT can be increased or decreased according to the distance between the two GHs. By drawing, undrawing, and bending the FT, the designer can obtain the intended shape [26]. Initially, the FT does not affect the shape of a curve. Once the FT becomes effective by pressing one $\mathrm{GH}$, the designer can alter the curve by pushing the FT against it (Fig. 12(a)). In addition, the designer can replace the deformable range of a curve with part of the FT (partial copying or patterning) [15]. The connection between two curve portions is determined by whether one or two GHs are clicked (Fig. 12(b) and (c)).

- Smooth: A rubbing gesture representing the action of ironing or sandpapering can be used to replace unwanted wiggles in a curve (Fig. 13).

Step 3. Fixate. Partial curve deformation is completed by selecting the curve portion outside the deform area and then clicking (Fig. 14).

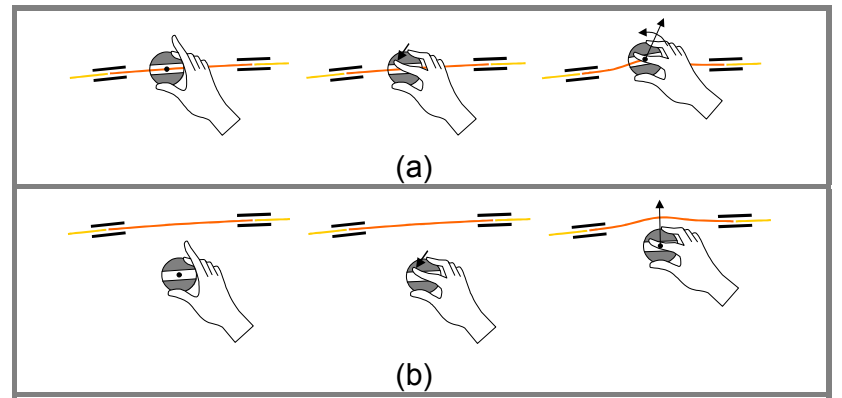

Figure 9: One-handed (a) direct and (b) indirect deformation 


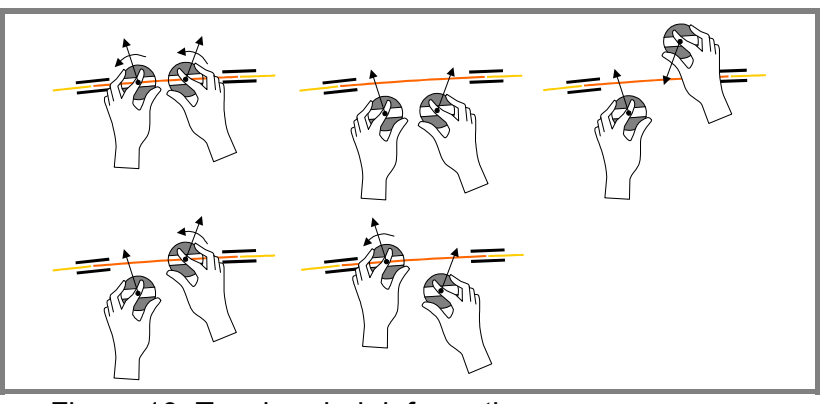

Figure 10: Two-handed deformations

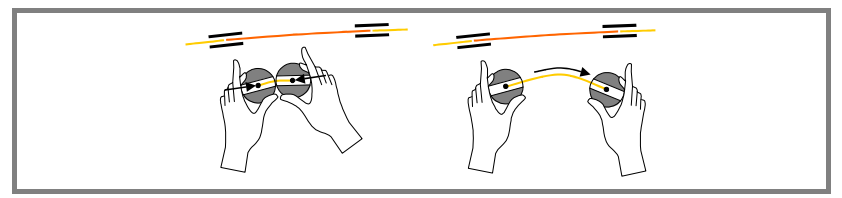

Figure 11: Generating a flexible template

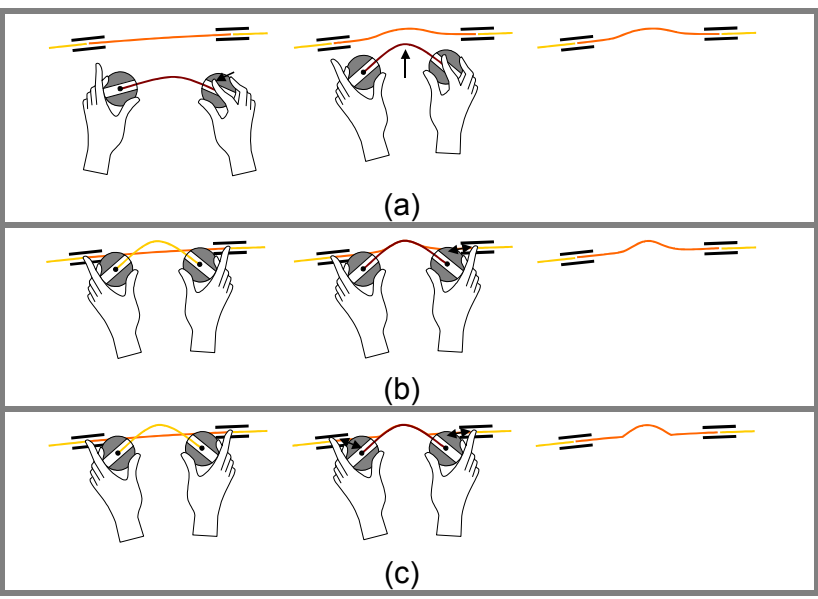

Figure 12: Three types of deformation with a flexible template

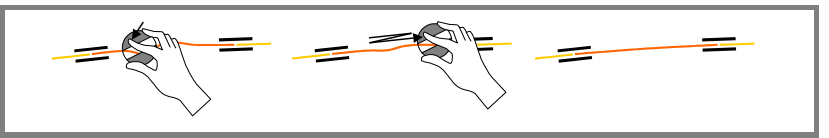

Figure 13: Smoothing

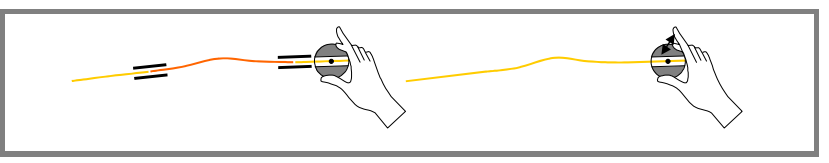

Figure 14: End of deformation

\section{Working Environment Control}

We integrated some important interaction ideas from previous studies into our prototype system to create a comfortable working environment and fluid user interaction.

- Two-handed zoom and pan [22]: Dragging the two GHs on the background of the screen is used for navigation. Moving the two GHs apart and together zooms the screen image in and out, respectively; moving in the same direction pans. These navigation functions are essential when handling small curve features with respect to the size of our GH.

- Marking menu [21]: Window controls, such as menus, icons, and buttons, which are generally located at fixed positions on the display screen, may divert the user's visual attention, especially in a large-display interaction environment. In our system, many support utilities (file in/out, grid on/off, curve evaluation measures on/off) are operated through pop-up menus that appear when and where the user wants.

\section{SOFTWARE IMPLEMENTATION}

We implemented the suggested interaction techniques using Java (J2SETM). Fig. 15 shows the relationships between our NURBS-curve manipulation operations.

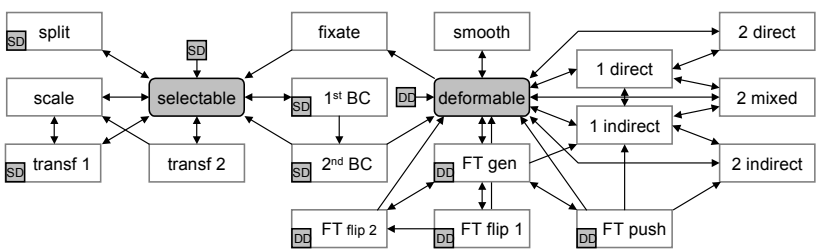

Figure 15: Operational flow between the NURBS-curve manipulations (SD: Selectable Dead, DD: Deformable Dead)

Despite our attempt to make successive user interactions seamless and fluid, some bad sequences of operations may occur. Imagine the situation involving imposing the first BC (refer to Fig. 8(a)); what if ND does the press before D completes the click? Therefore, we defined two dead states: selectable dead and deformable dead. If the user makes incorrect operations, the state of our system changes to the corresponding dead state and a message appears on the screen. The user can escape from the dead state by moving both GHs out of the down state.

\section{NURBS-curve Deformation Algorithm}

For a more realistic curve deformation result, the Finite Element Method (FEM) can be applied under the physical constraints imposed by the GHs (Fig. 16(a) and (b)), while assuming that a given NURBS curve is made of a real material. Since the user's curve-deform action is presumed smooth and monotonic and enough visual feedback is given [18], we chose a simple curve-deformation procedure using the constraint-based curve modification algorithm [12].

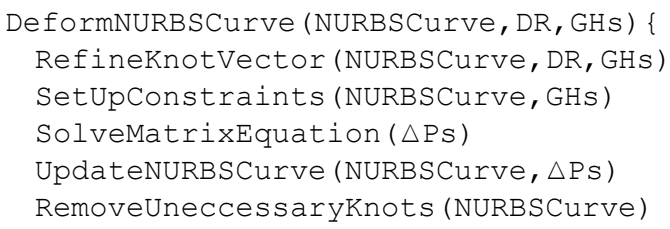

At each time step of the partial deformation of a NURBS curve, depending on the posture of each $\mathrm{GH}$ and its change, 
knots are added to the deform range (DR) of the curve to keep the portion outside the DR fixed, and to raise the degrees-of-freedom sufficiently for deformation. For direct modification, both the position and tangent constraints are assigned to the holding point of the curve; for indirect modification, only the position constraint is imposed on the closest point on the curve from the GH. After updating the NURBS curve based on the control-point changes $(\triangle \mathrm{Ps})$ that result from the constraint-based curve modification algorithm, all the unnecessary knots are removed. Fig. 16(c) and (d) show the incremental results of direct and indirect modifications, respectively.

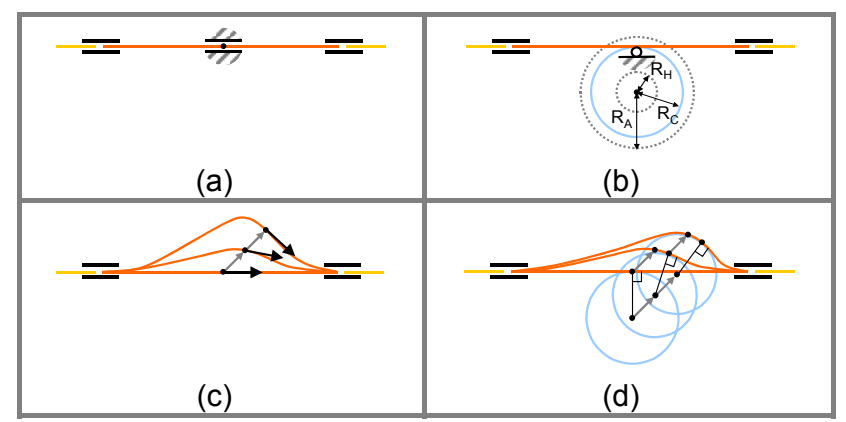

Figure 16: Physical models of (a) direct and (b) indirect deformation; incremental results of (c) direct and $(d)$ indirect deformation

\section{USER FEEDBACK AND DISCUSSION}

Six students majoring in art tested our prototype system. All of them showed great interest in being able to manipulate the virtual curves with simple handles. They readily accepted the holding and cutting metaphor of our $\mathrm{GH}$, and easily learned the basic operation and gesture set in minutes. Most of the test users became fully familiar with complex NURBS-curve operations within half an hour. The following key issues arose from the user feedback:

- Our vision-based tracking technique produced some jittering because the camera resolution was not sufficiently high compared with the size of the entire display screen $(2.4 \mathrm{~mm} / \mathrm{pixel})$ and the user's body caused optical noise. For stable tracking of our GHs, we regulated the signals based on averaging. Therefore, the users were aware of a time lag. We are now considering a multi-camera configuration to enhance spatial accuracy and PC cluster techniques to increase display speed.

- The confirmation level of the change of the DSVs was high because physical contact occurred, whereas that of the groove gestures was comparatively low, because the user had to depend on visual information only (the color and size of the GH cursor). Some users requested additional information. Feedback via sound can be added to the system easily, whereas providing physical cues, such as a bump or click, is somewhat problematic. If a small haptic feedback unit were embedded in the GH, or if new interactive media with force feedback [23,25,27] were introduced, the designer could manipulate curves with a more realistic feeling.
- The designer's hands or the GHs may partially obstruct the screen image while handling curves. In fact, this situation is very natural when manipulating physical material in real space. However, we do not think such obstructions will seriously decrease the working performance because our $\mathrm{GH}$ allows the user to see both the curve under consideration and the cursor of the GH through the transparent slit.

- We frequently observed users interchanging the functions of the right and left hands when performing two-handed curve deformations. This looked like empty-handed clay modeling and flexible curve handling. Owen et al. [24] mentioned that these tasks are visually and conceptually integrated reshaping activities. Even in this case, we think that the KC model is still valid because the role of each hand is determined spontaneously at every moment according to the relative positions of the hands and the object being deformed.

- One user used only a small portion of the screen by actively using pan and zoom functions. This observation led us to consider the focus-plus-context-screen display scheme [7], which offers comparatively small high-resolution regions and large low-resolution regions, and should help to reduce the load on the display server.

- Some users mentioned the ergonomics of the GHs. The $\mathrm{GH}$ presented here is a very early prototype that satisfies minimum functional requirements. Subsequent studies should examine its shape and usability. We are planning to make a malleable grip so that the user can seize it comfortably regardless of the orientation of the GH. In addition, we are considering scabbards for our $\mathrm{GH}$ because holding the GHs in both hands for a long time might prove uncomfortable.

- In a tabletop interactive system, many space-multiplexed input devices can be activated simultaneously by simply placing them on the horizontal display surface $[11,29]$. One of our test users suggested attachable markers made of suction cups for our vertical interaction surface (Fig. 17). A unique retro-reflective pattern on each attachable marker will deliver a specific meaning to the computer. One typical use is adding various geometric constraints during curve modification.

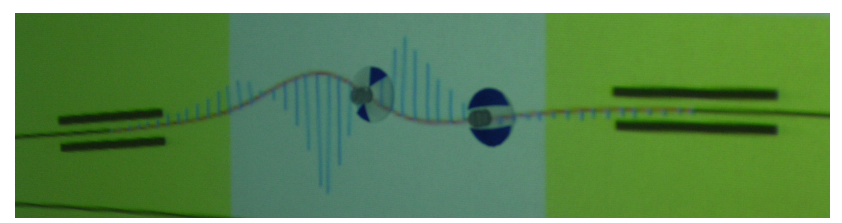

Figure 17: Attachable markers

- One test user stated that drawing and throwing would be more intuitive when splitting and deleting a curve, respectively (Fig. 18(a) and (b)). In addition, this user suggested counterclockwise and clockwise swirling gestures for undo and redo (Fig. 18(c) and (d)). We think that his curve-split gesture would be appropriate only for quick and rough cutting, because it contains no confirmation procedure for the exact cutting point. 


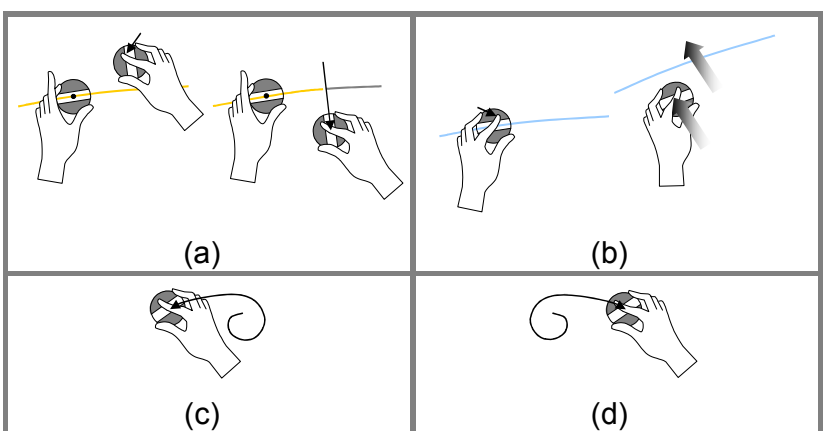

Figure 18: Suggested gestures for (a) curve-split, (b) curve-delete, (c) undo, and (d) redo

- For practical use, certain geometric and engineering constraints require the numeric parameters of a curve directly. FlowMenu [17,18], a fluid text input method for large displays, can be used for this purpose.

\section{CONCLUSIONS AND FUTURE WORK}

This paper describes a new digital system that allows designers to manipulate large-scale NURBS curves on a rigid interactive wall. We identified specific requirements for automotive design after considering ideal digital-design flow, and synthesized previous research to realize a novel digital-styling method that satisfies the design goals.

We developed a new GH with a transparent groove. Our GH enables the user to perform a holding action naturally - the most basic action when physically handling a curved shape in the real world. Despite its simple appearance, our GH is very expressive because the user's fingering motion can change its silhouette, which the computer recognizes. The user test showed that users readily accepted the basic on-screen gestures of our GH, which added a high level of tangibility to NURBS-curve manipulation techniques originating from traditional styling methods. We are confident that automotive designers will be able to exhibit their artistic abilities when modifying large-scale NURBS curves using our system.

We hope that our digital system will evolve into an essential tool for automotive designers through continuous enhancement and user testing, and that it will ultimately contribute to ubiquitous computing [30] in the field of automotive design. Future studies will improve its spatial accuracy and latency, examine the ergonomic design of the $\mathrm{GH}$, combine our method with flexible curve creation methods, derive additional interaction techniques for more delicate secondary curve operations, and establish a multi-user collaboration environment.

\section{ACKNOWLEDGMENTS}

The authors thank Eun-Jin Lee for fruitful discussion and for video production, Tovi Grossman for valuable advice, Jinah Park for editorial help, Andreas Schneider and IAMAS students for user feedback, and all the reviewers for helpful comments.

\section{REFERENCES}

1. Arvo, J. and Novins, K. Fluid sketches: continuous recognition and morphing of simple hand-drawn shapes. In Proceedings of UIST 2000, pp. 73-80.

2. Bae, S.-H., Kim, W.-S. and Kwon, E.-S. Digital styling for designers: sketch emulation in computer environment. LNCS, Vol. 2669, 2003, pp. 690-700.

3. Bae, S.-H. and Kijima, R. Digital styling for designers: in prospective automotive design. In Proceedings of VSMM 2003, pp. 546-553.

4. Balakrishnan, R., Fitzmaurice, G., Kurtenbach, G. and Buxton, W. Digital tape drawing. In Proceedings of UIST 1999, pp. 161-169.

5. Balakrishnan, R., Fitzmaurice, G., Kurtenbach, G. and Singh, K. Exploring interactive curve and surface manipulation using a bend and twist sensitive input strip. In Proceedings of I3DG 1999, pp. 111-118.

6. Baudel, T. A mark-based interaction paradigm for free-hand drawing. In Proceedings of UIST 1994, pp. 185-192.

7. Baudisch, P., Good, N. and Stewart, P. Focus plus context screens: combining display technology with visualization techniques. In Proceedings of UIST 2001, pp. 31-40.

8. Buxton, W. and Myers, B.A. A study in two-handed input. In Proceedings of CHI 1986, pp. 321-326.

9. Buxton, W., Fitzmaurice, G., Balakrishnan, R. and Kurtenbach, G. Large displays in automotive design. IEEE CG\&A, July/Aug 2000, pp. 68-75.

10. Elrod, S., Bruce, R., Gold, R., Goldberg, D., Halasz, F., Janssen, W., Lee, D., McCall, K., Pedersen, E., Pier, K., Tang, J. and Welch, B. LiveBoard: a large interactive display supporting group meetings, presentations and remote collaboration. In Proceedings of CHI 1992, pp. 599-607.

11. Fitzmaurice, G.W., Ishii, H. and Buxton, W. Bricks: laying the foundations for graspable user interfaces. In Proceedings of CHI 1995, pp. 442-449.

12. Fowler, B. and Bartels, R. Constraint based curve manipulation. IEEE $C G \& A$, Vol. 13, No. 5, 1993, pp. 43-49.

13. Grossman, T., Balakrishnan, R., Kurtenbach, G., Fitzmaurice, G., Khan, A. and Buxton, B. Interaction techniques for $3 \mathrm{D}$ modeling on large displays. In Proceedings of I3DG 2001, pp. 17-23.

14. Grossman, T., Balakrishnan, R., Kurtenbach, G., Fitzmaurice, G., Khan, A. and Buxton, B. Creating 
principal 3D curves with digital tape drawing. In Proceedings of CHI 2002, pp. 121-128.

15. Grossman, T., Balakrishnan, R. and Singh, K. An interface for creating and manipulating curves using a high degree-of-freedom curve input device. In Proceedings of CHI 2003, pp. 185-192.

16. Guiard, Y. Asymmetric division of labour in human skilled bimanual action: the kinematic chain as a model. J. of Motor Behavior, Vol. 19, 1987, pp. 486-517.

17. Guimbretière, F. and Winograd, T. FlowMenu: combining command, text, and parameter entry. In Proceedings of UIST 2000, pp 213-216.

18. Guimbretière, F., Stone, M. and Winograd, T. Fluid interaction with high-resolution wall-size displays. In Proceedings of UIST 2001, pp. 21-30.

19. Igarashi, T., Matsuoka, S. and Tanaka, H. Teddy: a sketching interface for 3D freeform design. In Proceedings of SIGGRAPH 1999, pp. 409-416.

20. Kabbash, P., Buxton, W. and Sellen, A. Two-handed input in a compound task. In Proceedings of CHI 1994, pp. 417-423.

21. Kurtenbach, G. and Buxton, W. The limits of expert performance using hierarchic marking menus. In Proceedings of INTERCHI 1993, pp. 482-487.

22. Kurtenbach, G., Fitzmaurice, G., Baudel, T. and Buxton, B. The design of a GUI paradigm based on tablets, two-hands, and transparency. In Proceedings of $\mathrm{CHI}$ 1997, pp. 35-42.

23. Noma, H., Yanagida, Y. and Tetsutani, N. The proactive desk: a new force display system for a digital desk using 2-DOF linear induction motor. In Proceedings of IEEE VR2003, pp. 217-224.
24. Owen, R., Kurtenbach, G., Fitzmaurice, G., Baudel, T. and Buxton, W. Bimanual manipulation in a curve editing task. Unpublished Manuscript, Alias|Wavefront Inc. 1998, http://www.alias.com/eng/about/research/ papers/index.shtml.

25. Pangaro, G., Maynes-Aminzade, D. and Ishii, H. The actuated workbench: computer-controlled actuation in tabletop tangible interfaces. In Proceedings of UIST 2002, pp. 181-190.

26. Rekimoto, J. and Matsushita, N. Perceptual surfaces: towards a human and object sensitive interactive display. In Proceedings of PUI 1997, pp. 30-32.

27. Rekimoto, J. SmartSkin: an infrastructure for freehand manipulation on interactive surfaces. In Proceedings of CHI 2002, pp. 113-120.

28. Singh, K. Interactive curve design using digital French curves. In Proceedings of I3DG 1999, pp. 23-30.

29. Ullmer, B. and Ishii, H. The metaDESK: models and prototypes for tangible user interfaces. In Proceedings of UIST 1997, pp. 223-232.

30. Weiser, M. The computer for the 21 st century. Scientific American, Vol. 265, No. 3, 1991, pp. 94-104.

31. Wellner, P. Interacting with paper on the DigitalDesk. Com. of ACM, Vol. 36, No. 7, 1993, pp. 86-96.

32. Wu, M. and Balakrishnan, R. Multi-finger and whole hand gestural interaction techniques for multi-user tabletop displays. In Proceedings of UIST 2003, pp. 193-202.

33. Yamada, Y. Clay Modeling: Techniques for Giving Three-dimensional Form to Idea, Car Styling Extra Issues, Vol. 93 1/2, 1993. 